\title{
Influence of substrate and film thickness on polymer LIPSS formation
}

\author{
Jing Cui ${ }^{1}$, Aurora Nogales ${ }^{1}$, Tiberio A. Ezquerra ${ }^{1}$, Esther Rebollar ${ }^{2 *}$ \\ ${ }^{1}$ Instituto de Estructura de la Materia (IEM-CSIC), Serrano 121, Madrid, 28006.Spain \\ ${ }^{2}$ Instituto de Química Física Rocasolano (IQFR-CSIC), Serrano 119, Madrid, 28006. Spain \\ *Corresponding author: e.rebollar@csic.es
}

\begin{abstract}
Here we focus on the influence of both, substrate and film thickness on polymer Laser Induced Periodic Surface Structures (LIPSS) formation in polymer films. For this aim a morphological description of ripples structures generated on spin-coated polystyrene (PS) films by a linearly polarized laser beam with a wavelength of $266 \mathrm{~nm}$ is presented. The influence of different parameters on the quality and characteristics of the formed laserinduced periodic surface structures (LIPSS) was investigated. We found that well-ordered LIPSS are formed either on PS films thinner than $200 \mathrm{~nm}$ or thicker than $400 \mathrm{~nm}$ supported on silicon substrates as well as on thicker free standing films. However lessordered ripples are formed on silicon supported films with intermediate thicknesses in the range of 200-380 $\mathrm{nm}$. The effect of substrate on the quality of LIPSS was analyzed in terms of its thermal and optical properties. Differences observed in the fluence and number of pulses needed for the onset of surface morphological modifications is explained considering two main effects which are: 1) The temperature increase on polymer surface induced by the action of cumulative laser and 2) The differences in thermal conductivity between the polymer and the substrate which strongly affect the heat dissipation generated by irradiation.
\end{abstract}

Keywords: Laser-induced periodic surface structures; Polystyrene; Film thickness; Substrates properties; Pulse accumulation; 


\section{INTRODUCTION}

Controlling the surface morphology over multiple length scales, ranging from micro- to nanometers, can be a key factor to optimize materials performance for certain applications [1] and to obtain tailor made properties [2-4]. As an example, frictional, adhesive, and wetting forces of a material surface can be strongly influenced by its micro- and nanoscale structure [5]. Surface nanostructures in polymers have been obtained by several methods mostly based on advanced soft lithography [6-8]. Laser techniques are very versatile alternative for nanostructuring since the use of clean rooms, high vacuum, or mask fabrication is not necessary. In addition, they can be applied to different polymer materials in large/mesoscopic areas [9-11]. In particular, Laser Induced Periodic Surface Structures (LIPSS) on polymers can be obtained by using intense laser pulses. This technique can be considered as a potential alternative strategy to lithography processes, for obtaining functional nanopatterned polymer surface structures [12-14]. LIPSS have been produced on different materials using lasers with pulse duration ranging from nanoto femtoseconds and wavelengths from the IR to the UV spectral regions [15-18]. To obtain LIPSS, the polymer surface is exposed to a given number of pulses of a linearly polarized laser beam within a narrow fluence range well below the ablation threshold [19-21]. The period of the obtained ripples $(L)$ is related to the laser wavelength $(\lambda)$, the effective refractive index $(n)$ of the material, and the incidence angle $(\theta)$ of the laser beam with respect to the normal to the sample plane by the following expression [22]:

$$
L=\frac{\lambda}{n-\sin \theta}
$$

The mechanism of ripple formation on polymer surfaces is complex and still not completely well understood. It is widely accepted that LIPSS formed on the material surface originates from the interference between the incident and the surface scattered waves, which induced a heterogeneous intensity distribution, and together with a feedback mechanism results in the enhancement of the modulation intensity $[8,13,19,23]$. It is important to note that ripples formation is originated from a self-organization process and not caused by ablation, being the amount of material removed during laser irradiation negligible [16]. During LIPSS formation, two main stages can be considered: (i) the formation of an interference pattern at the surface, and (ii) the surface response to it. The features of LIPSS might be affected by the polymer properties, which are dependent not only on the chemical composition, but also on the polymer state, amorphous or semicrystalline, and on the hierarchical structure of the crystalline phase. Processes like thermal and nonthermal scissoring of polymer chains, amorphization, local surface melting, ablation, photooxidation, material transport and rearrangement, have been proposed to be involved in ripple formation [14, 19, 21, 24, 25]. More recently it has been reported that when a polymer film is irradiated with a laser beam at a wavelength efficiently absorbed, the surface 
temperature increases. Thus, in order to obtain LIPSS, the temperature should be high enough to grant significant polymer chain mobility. In particular, temperature should be either above the glass transition temperature $\left(\mathrm{T}_{\mathrm{g}}\right)$ for the case of amorphous polymer, allowing polymer chains to rearrange $[12,13,26]$, or above its melting temperature $\left(T_{m}\right)$ in the case of a semicrystalline polymer so that melting of the surface crystallites occurs providing enough polymer dynamics [27] and the redistribution of the material [28]. In general, LIPSS can be prepared in both spincoated [27, 29] and in free standing polymer films [26], which makes LIPSS a potential method to obtain large processed surface area and good quality samples. Properties of the nanostructured polymers will be relevant in order to develop applications. Together with the morphological changes induced upon laser irradiation, chemical changes can take place. In particular it has been reported for several polymers that oxygen concentration can change upon UV irradiation in air $[18,30,31]$. Surface induced changes also provoke modifications in wettability $[32,33]$ which are of great importance for applications such as improving the adhesion of coatings [34-36], preparation of nanowires [36, 37], and cell proliferation [18] and alignment [38].

Film thickness may play also an important role on LIPSS formation. Csete et al observed [28] that linear LIPSS are formed in films with thickness above a minimum thickness value. Thus, LIPSS can be obtained for free-standing films with thicknesses in the range of microns while for supported films thinner than 60 nanometers the line-shaped LIPSS structures are transformed into droplets, due to laser induced polymer melting and subsequent dewetting from the substrate. Also substrate nature may affect LIPSS properties as reported by Kalachyova et al. [39] for doped poly(methyl methacrylate).

Despite the number of works published on the thermal evolution during LIPSS formation $[13,19,40]$, several key issues remain unclear. Among these: 1 ) how does the temperature evolve during pulse accumulation on the polymer surface? 2) Does the underlying substrate have any influence on LIPSS formation? and 3) What is the role of film thickness on optimum LIPSS formation?

The main aim of this work is to answer these questions by relating the quality of the ripples induced by multipulse laser irradiation on polymer films with the thickness of the film, substrate properties, and number of pulses of the irradiation.

\section{EXPERIMENTAL}

\subsection{Sample preparation}

Polystyrene (PS), from Polymer Source, with number molecular weight $\left(\mathrm{M}_{\mathrm{n}}\right)$ of 51,000 $\mathrm{g} / \mathrm{mol}$ and PDI 1.05 , was used as received. PS is an amorphous polymer, with glass transition 
temperature $\mathrm{T}_{\mathrm{g}}=105^{\circ} \mathrm{C}$. Silicon wafers (100) (Wafer World Inc.), glass (microscope slides, Thermo Scientific) and quartz substrates (UQG Optics) were cut into pieces of $1 \times 1 \mathrm{~cm}^{2}$. All the substrates were first cleaned by chloroform (99.98\% purity, QUIMIPUR-Spain). Supported PS thin films of various thicknesses, depending on the polymer concentration, were obtained by spin coating chloroform polymer solutions on different substrates, with a rotation speed of $2380 \mathrm{rpm}$ during 2 min. Thickness was measured by atomic force microscopy (AFM). Roughness of the spin coated films was also measured by AFM at three different locations of each film. Details of the characteristics of the prepared films can be found in Table 1. Thick PS free-standing films were prepared by casting PS solution onto selected substrates, dried in vacuum oven for one week, and then removed from the substrate. The thickness was estimated to be around $150 \times 10^{3} \mathrm{~nm}$ by using a micrometer caliper.

Table 1. Characteristics of the prepared PS films.

\begin{tabular}{|c|c|c|c|c|}
\hline Substrate & $\begin{array}{c}\text { Concentration } \\
(\mathbf{g} / \mathbf{L})\end{array}$ & $\begin{array}{c}\text { Thickness } \\
\mathbf{( n m})\end{array}$ & $\begin{array}{c}\text { Roughness } \\
\mathbf{( n m})\end{array}$ & Sample Label \\
\hline \multirow{3}{*}{ Silicon } & 8 & $67 \pm 3$ & $0.22 \pm 0.01$ & Si08 \\
\cline { 2 - 5 } & 10 & $132 \pm 5$ & $0.23 \pm 0.01$ & Si10 \\
\cline { 2 - 5 } & 15 & $231 \pm 8$ & $0.27 \pm 0.01$ & Si15 \\
\cline { 2 - 5 } & 20 & $278 \pm 12$ & $0.29 \pm 0.01$ & Si20 \\
\cline { 2 - 5 } & 25 & $388 \pm 15$ & $0.39 \pm 0.01$ & Si25 \\
\cline { 2 - 5 } & 30 & $434 \pm 10$ & $0.34 \pm 0.02$ & Si30 \\
\hline \multirow{7}{*}{ Glass } & 10 & $115 \pm 15$ & $0.37 \pm 0.01$ & G10 \\
\cline { 2 - 5 } & 20 & $244 \pm 21$ & $0.30 \pm 0.01$ & G20 \\
\hline Quartz & 10 & $106 \pm 9$ & $0.21 \pm 0.01$ & Q20 \\
\cline { 2 - 5 } & 20 & $261 \pm 13$ & $0.38 \pm 0.01$ & PS60 \\
\hline PS casting & 60 & $(150 \pm 7) \times 10^{3}$ & $0.39 \pm 0.02$ & \\
\hline
\end{tabular}

The absorption spectrum of a PS film was measured by a UV-Vis-NIR spectrophotometer (UV3600 Shimadzu), in the wavelength range from 1100 to $200 \mathrm{~nm}$. The linear absorption coefficient of the used PS is $1.6 \times 10^{5} \mathrm{~m}^{-1}$ at $266 \mathrm{~nm}$.

\subsection{Laser Irradiation}

Laser irradiation was carried out in ambient air under normal incidence, with the linearly polarized laser beam of a Q-switched Nd:YAG laser (Lotis TII LS-2131 M, pulse duration $\tau=8$ ns full 
width half-maximum) at a repetition rate of $10 \mathrm{~Hz}$. The fourth harmonic, with a wavelength $\lambda=$ $266 \mathrm{~nm}$ was used for the generation of LIPSS on PS. The irradiation fluence was determined by measuring the laser energy in front of the sample with a joulemeter (Gentec-E, QE25SP-H-MBD0) and by calculating the area of the irradiated spots after delimitating the beam with an iris of $0.29 \mathrm{~cm}$ diameter. Polymer films were irradiated under various pulses (up to several thousands) at a constant fluence. In particular cases, the influence of the fluence at fixed number of pulses were also studied.

\subsection{Morphology characterization}

Topography of the PS films before and after irradiation was characterized by Atomic Force Microscopy (AFM). A multimode 8 AFM equipped with a Nanoscope V Controller by Bruker, with selected probe NSG30 (NT-MDT) in tapping mode was employed. AFM images were analyzed with the software Nanoscope Analysis 1.50 (Bruker). The periods and heights of formed LIPSS were determined from the AFM analysis by averaging the values obtained at 3 different areas of the irradiated sample.

\section{RESULTS AND DISCUSSION}

\subsection{Effect of the number of pulses on LIPSS formation}

In order to better understand the effect of varying the number of pulses $(N)$ on the quality of the formed LIPSS patterns, PS films were irradiated at a fixed fluence $F=10 \mathrm{~mJ} / \mathrm{cm}^{2}$ with a repetition rate of $10 \mathrm{~Hz}$ varying up to a maximum $N$ of 6000 . AFM height images of the silicon supported thin films as a function of $N$ are shown in Figure 1 for the sample Si30 with a thickness of $434 \pm$ $10 \mathrm{~nm}$.
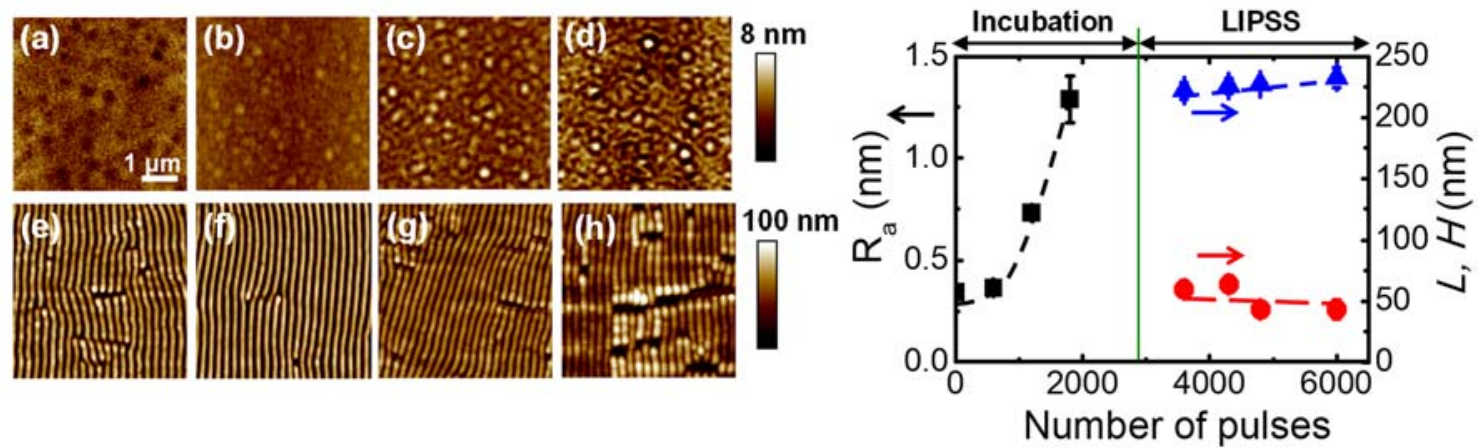

Figure 1. (Left panel) AFM height images $\left(5 \times 5 \mu \mathrm{m}^{2}\right)$ of for Si30 (434 nm) (a) non-irradiated and irradiated at $266 \mathrm{~nm}$ with a fluence of $10 \mathrm{~mJ} / \mathrm{cm}^{2}$ with (b) 600, (c) 1200, (d) 1800, (e) 3600, (f) 4300, (g) 4800, and (h) 6000 pulses. (Right panel) Average roughness (Ra) (black squares), Period ( $L$, blue triangles) and depth 
( $H$, red circles) as a function of number of laser pulses for Si30 irradiated at constant $10 \mathrm{~mJ} / \mathrm{cm}^{2}$. The dashed lines are plotted to guide the eye.

As observed in Figure 1, to obtain LIPSS in the surface of the polymer film, it is necessary to irradiate with a relative large number of pulses. The periodic surface structures are not formed until the number of pulses is $\approx 3600$. However, before the LIPSS are formed, irradiation with a low number of pulses affects the roughness of the PS surface (Figure 1b and Figure 1c). Figure 1 (right panel) shows that the surface roughness increases in the time period previous to LIPSS formation, referred to hereafter as incubation regime, and the values of both period and depths of the LIPSS.

As previously reported $[9,13]$, when a polymer film is exposed to the laser light, the film surface scatters light due to its roughness and these scattered waves interfere with the incident laser beam resulting in a modulated distribution of the energy on the surface [13]. This energy induces a modulated change in temperature in such a way that when this temperature is higher than the glass transition temperature of the irradiated polymer, a softening of the outermost layer occurs, allowing diffusion of the polymeric chains [41].

As shown in Figure 1, repetitive irradiation produces changes in the topography of the polymer surface and ripples morphology develops after a sufficient number of laser pulses have arrived to the polymer surface. Considering laser irradiation as the only heating source of the polymer film, the corresponding temperature increase can be estimated by solving the one-dimensional heat equation [13]. Thus, the temperature at a given time $(t)$ and depth $(x)$ from the surface should follow the equation $[13,19]$ :

$$
\frac{\partial^{2} T(x, t)}{\partial x^{2}}-a^{2} \frac{\partial T(x, t)}{\partial t}=-\frac{\alpha}{\kappa} P(t) e^{-\alpha x} F_{0}
$$

Where $a^{2}=\frac{\rho c}{\kappa}$ being $\rho$ the density, $c$ the specific heat at constant pressure, $\kappa$ the thermal conductivity, and $\alpha$ the absorption coefficient. $F_{0}$ is the incident fluence, being $10 \mathrm{~mJ} / \mathrm{cm}^{2}$ in this work, and $P(t)$ is a function which describes the temporal shape of the laser pulse which is considered as a modified Gaussian beam:

$$
P(t)=\frac{2 t}{\tau^{2}} \exp \left[-(t / \tau)^{2}\right]
$$

Where the $t$ factor ensures that intensity vanishes at $t=0$ and $\tau$ is the pulse duration, in our case 8 ns. Using the appropriated Green's function, $\mathrm{T}(x, t)$ can be calculated. For the calculations an initial temperature of $23{ }^{\circ} \mathrm{C}$ and the values of the physical properties reported in Table 2 have been considered. 
Table 2. Linear absorption coefficient $(\alpha)$ at $266 \mathrm{~nm}$, specific heat $(c)$, density $(\rho)$, thermal conductivity $(\kappa)$, and refractive index at $266 \mathrm{~nm}(n)$ of PS and of the supporting substrates.

\begin{tabular}{ccccc} 
& PS & Silicon & Quartz & Glass \\
\hline $\boldsymbol{\alpha}\left(\mathbf{m}^{-\mathbf{1}}\right)$ & $1.6 \times 10^{5}$ & $1.97 \times 10^{8}[42,43]$ & $3[44]$ & $\sim 10^{8}$ \\
$\boldsymbol{c}\left(\mathbf{J} \cdot \mathbf{K g}^{-1} \cdot \mathbf{K}^{-1}\right)$ & $1223[45]$ & $703[46]$ & $710[47]$ & $858[48]$ \\
$\boldsymbol{\rho}\left(\mathbf{K g} \cdot \mathbf{m}^{-3}\right)$ & $1043[41]$ & $2330[46]$ & $2649[47]$ & $2510[48]$ \\
$\boldsymbol{\kappa}\left(\mathbf{W} \cdot \mathbf{m}^{-\mathbf{1}} \cdot \mathbf{K}^{-1}\right)$ & $0.11[45]$ & $163.3[46]$ & $10.7[47]$ & $1.11[48]$ \\
$\boldsymbol{n}$ & $1.49[49]$ & $1.9[42,50]$ & $\sim 1.5[51]$ & $1.51[52]$ \\
\hline
\end{tabular}

For a single pulse, the variation of the calculated temperature at the surface as a function of the time elapsed from the beginning of the pulse is presented in Figure 2a. The result shows that, once the first pulse reaches the film, the temperature of the surface increases since the laser energy absorbed by PS is transformed into heat. As the pulse finishes, the surface of the sample starts to cool down due to the dissipation of heat into the cooler surrounding bulk material. The highest temperature achieved at the polymer surface by the initial single pulse is estimated to be around $32^{\circ} \mathrm{C}$, far below the $\mathrm{T}_{\mathrm{g}}$ of PS which is $105^{\circ} \mathrm{C}$. Although at first glance it seems that the temperature for long time reaches the initial one, a small but positive offset is still present at the time the next pulse arrives ( $0.1 \mathrm{~s}$ later than the onset of the previous one). Accumulation of laser pulses on the irradiated spot induces subsequent cycles of heating and cooling. Assuming that none of the physical magnitudes of the polymer change with temperature, one can estimate that each pulse will heat the sample and that the surface will be at a slightly higher temperature than the previous one after the arrival of the next laser pulse. This effect is illustrated in Figure $2 \mathrm{~b}$. A minimum number of pulses is therefore necessary to ensure that the surface temperature overcomes the $T_{g}$ of the polymer. Thus, with this approximate estimation of the temperature increment, this number of pulses is estimated to be, in our case, larger than 200. This result suggests that the increase on roughness observed in Figure 1 (right panel) during the incubation regime before LIPSS formation occurs when the temperature has increased above the $\mathrm{T}_{\mathrm{g}}$ of PS. In fact, for the sample Si30 slight changes in roughness are observed by AFM inspection already after irradiation with 450 pulses. After laser induced local heating, the material cools down and surface inhomogeneities are expected to be enhanced as the next pulse would find a rougher surface, thus facilitating the feedback mechanism needed for LIPSS formation [53]. It should be contemplated that here we performed a simplified temperature calculation where the temperature dependence of the polymer physical parameters was not considered. Additionally, the possible changes in the thermal properties of the surface during the transformations caused by the previous laser pulses (i.e., incubation effects) were also neglected. Nevertheless, considering only the thermal effects induced by every laser pulse and the repetitive heating/cooling cycles the calculation can explain 
LIPSS formation and supports the presence of the feedback mechanism

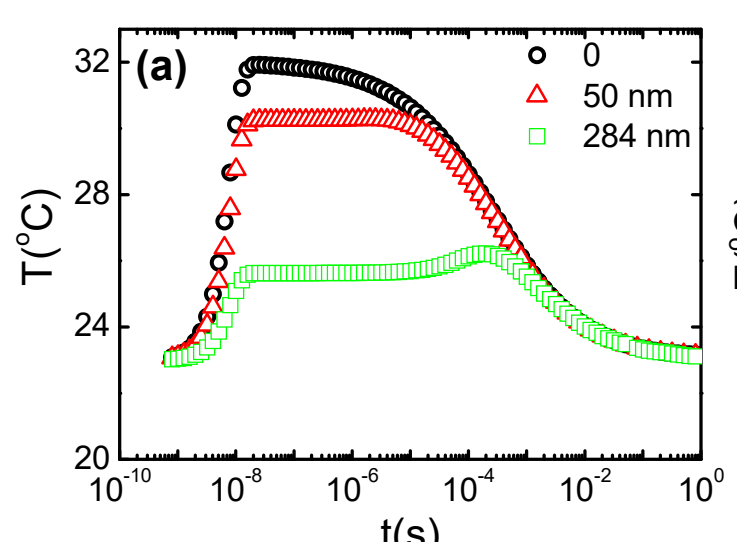

$\mathrm{t}(\mathrm{s})$

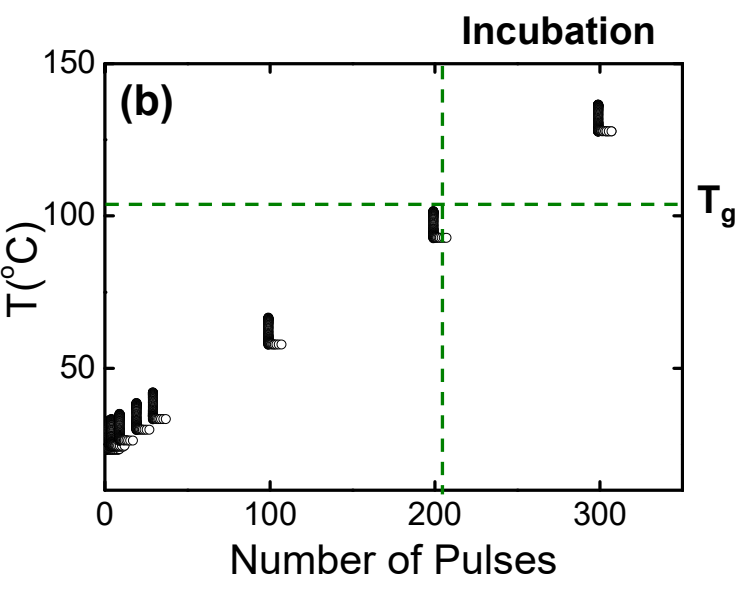

Figure 2. (a) Calculated time dependence of the surface temperature of PS after irradiation with the first pulse at $266 \mathrm{~nm}$ at a fluence of $10 \mathrm{~mJ} / \mathrm{cm}^{2}$ at the indicated depths. (b) Temperature estimation of polymer surface upon irradiation with successive laser pulses. Dashed line corresponds to the $\mathrm{T}_{\mathrm{g}}$ of PS.

\subsection{Influence of thickness on LIPSS formation}

To assess the influence of film thickness in the LIPSS formation, PS thin films with thickness values ranging from $70 \mathrm{~nm}$ to $150 \times 10^{3} \mathrm{~nm}$ were irradiated at fixed values of fluence, $10 \mathrm{~mJ} / \mathrm{cm}^{2}$, and number of pulses, $N=4800$. Figure 3 shows the corresponding AFM topography images. For the thinnest film, Si08 (Figure 3a), disordered LIPSS are formed. This is in agreement with previous results by Csete et al. [30] reporting about the existence of a critical thickness below which line-shaped structure cannot be formed. In contrast for thicker films, Si10 (Figure 3b), Si30 (Figure 3e) and PS60 (Figure $3 \mathrm{f}$ ), parallel ripples are observed. Curiously enough, in the case of films with intermediate thicknesses, Si15 (Figure 3c), Si20 (Figure 3d) and Si25 (not shown), distorted LIPSS decorated with droplet-like entities can be observed. The thickness dependence of the period and depth of LIPSS is also displayed in Figure $3 \mathrm{~g}$ and Figure $3 \mathrm{~h}$. The period and depth values of the obtained LIPSS in Si10, Si30 and PS60 are similar (around $220 \mathrm{~nm}$ and 50 $\mathrm{nm}$ respectively). In contrast, films with intermediate thickness exhibit larger average period, around $270 \mathrm{~nm}$, and deeper structures. From the above results it is possible to conclude that, for silicon supported thin films, a critical range of film thicknesses exist at which the formation of well-ordered LIPSS is hindered. 

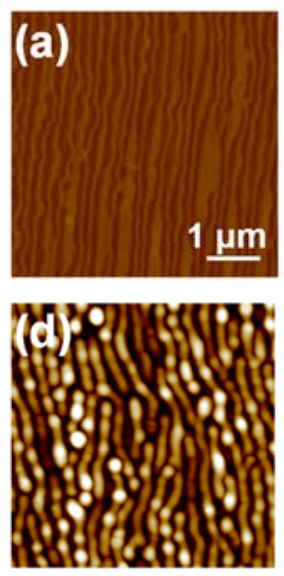
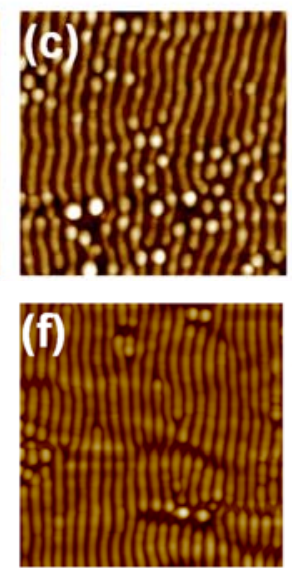

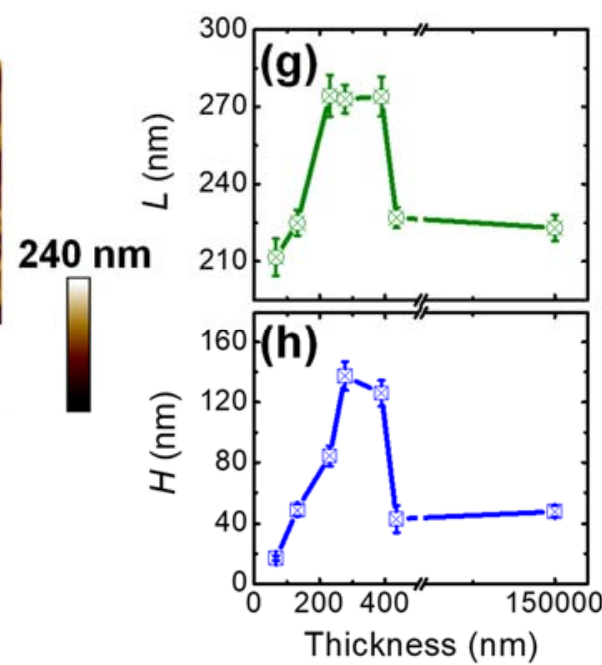

Figure 3. (Left) AFM height images $\left(5 \times 5 \mu^{2}\right)$ of PS films with different thicknesses: (a) Si08 (67 nm), (b) Si10 (132 nm), (c) Si15 (231 nm), (d) Si20 (278 nm), (e) Si30 (434 nm and (f) PS60 (150x10 nm). (Right) (g) Period length and (h) depth of the LIPSS as a function of the film thickness for PS film. The films were irradiated at a fluence of $10 \mathrm{~mJ} / \mathrm{cm}^{2}$ and number of pulses of 4800 . Lines are plotted to guide the eyes.

The variation of the average roughness $R_{a}$ with the number of pulses in the incubation regime for samples with different thicknesses is shown in Figure 4a. The roughness starts increasing slowly for a low number of pulses, and at a given value of $N$, around 600 in this case, the surface starts to corrugate. Changes are more significant for the film, Si30 (434 $\pm 10 \mathrm{~nm}$ thick), in comparison to thinner films.
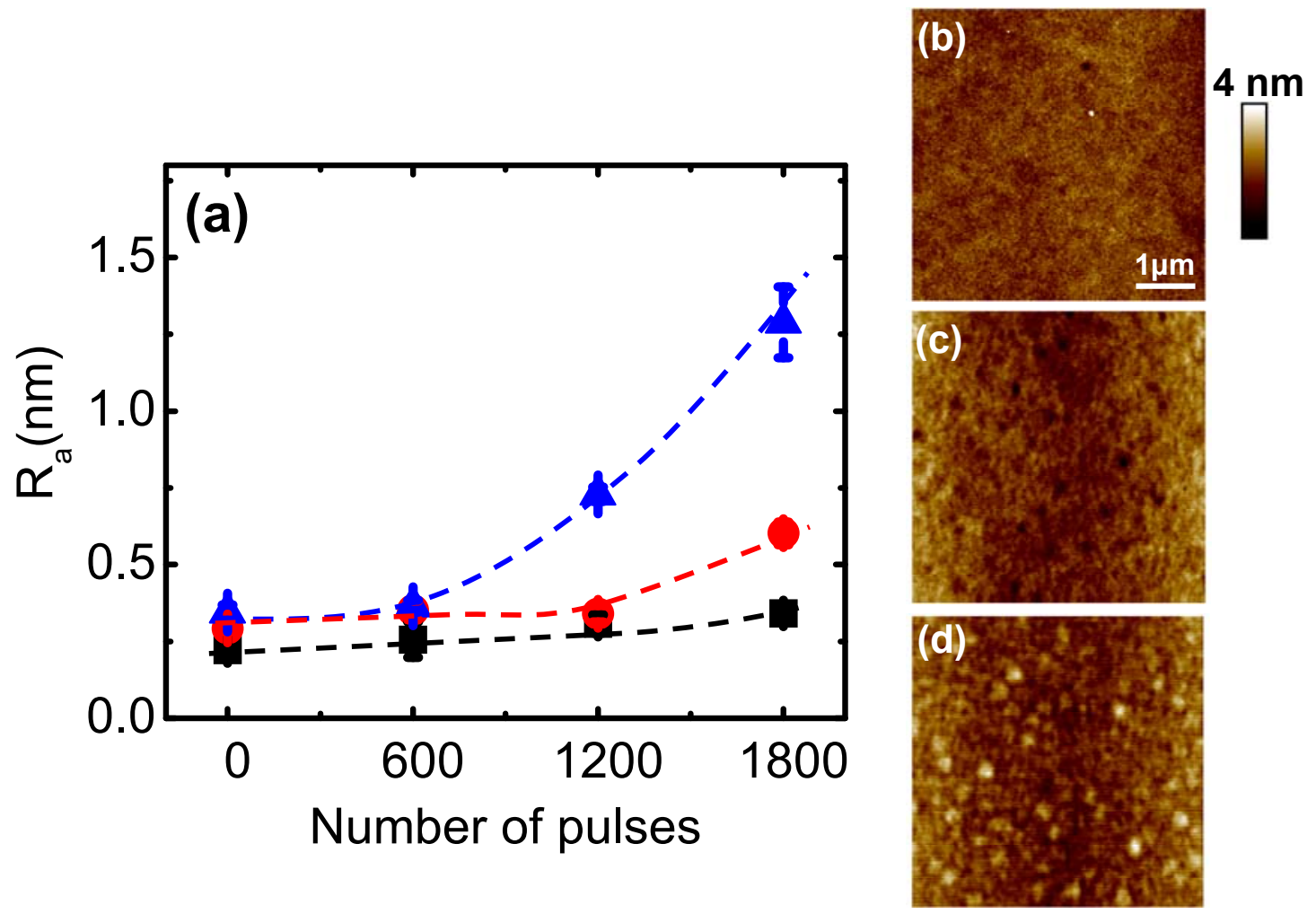
Figure 4. (a) Average roughness $\left(\mathrm{R}_{\mathrm{a}}\right)$ as a function of number of laser pulses for $\mathrm{Si10}(132 \pm 5 \mathrm{~nm}$ thick)

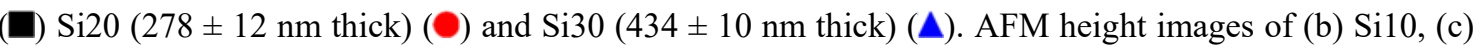
Si20 and (d) Si30 irradiated at constant $10 \mathrm{~mJ} / \mathrm{cm}^{2}$ and 600 pulses. Lines are a guide to the eye.

For a given sample, once well-defined LIPSS are formed, the period and depths have values around $220 \mathrm{~nm}$ and $50 \mathrm{~nm}$ respectively except for the case of Si20 for which both values are larger, in particular around $270 \mathrm{~nm}$ and $120 \mathrm{~nm}$ respectively. The differences observed for the polymer films with different thicknesses in terms of modification onset and LIPSS properties cannot simply be explained by considering exclusively the heating/cooling effects induced by repetitive irradiation discussed in the previous paragraph. Hence, the influence of the silicon substrate will be discussed in the following section. As it will be shown, specifically for silicon substrates, the laser irradiation of the PS films of different thickness provokes the substrate temperature to increase very fast (Table 3). However, the heat is effectively dissipated on the basis of the high thermal diffusivity of silicon (Table 3). Thus, the high thermal conductivity and thermal diffusivity of silicon makes the cooling of the polymer material heated by the laser pulse (see paragraph 3.1) to be more efficient for thinner films. Accordingly, the silicon substrate acts as a thermal heat sink making that thinner films need more irradiation energy, either in terms of pulses of in term of fluence, in order to reach similar temperature values than those reached by thicker films [54]. In our case, a thin film like Si10 $(132 \mathrm{~nm})$ needs a higher number of pulses than a thicker one in order to exhibit morphological changes as shown in Figure 4a. To further corroborate this effect additional irradiations varying laser fluence were performed. In this case, two films with different thicknesses, Si10 $(132 \mathrm{~nm})$ and Si20 $(278 \mathrm{~nm})$ were irradiated at different fluences, 7 and $12 \mathrm{~mJ} / \mathrm{cm}^{2}$. Figure 5 shows the corresponding AFM topographic images. In the case of the thinner film, Si10, irradiation with $7 \mathrm{~mJ} / \mathrm{cm}^{2}$ does not induce any significant morphological change (Figure 5a). However, an evident change in roughness can be detected for $\mathrm{Si} 20$ at the same irradiation conditions (Figure 5d). 

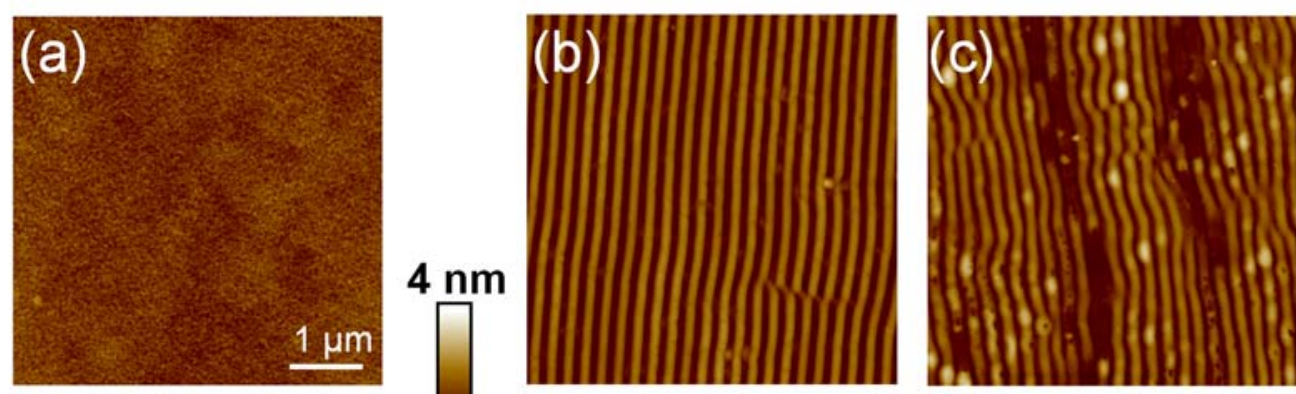

$180 \mathrm{~nm}$
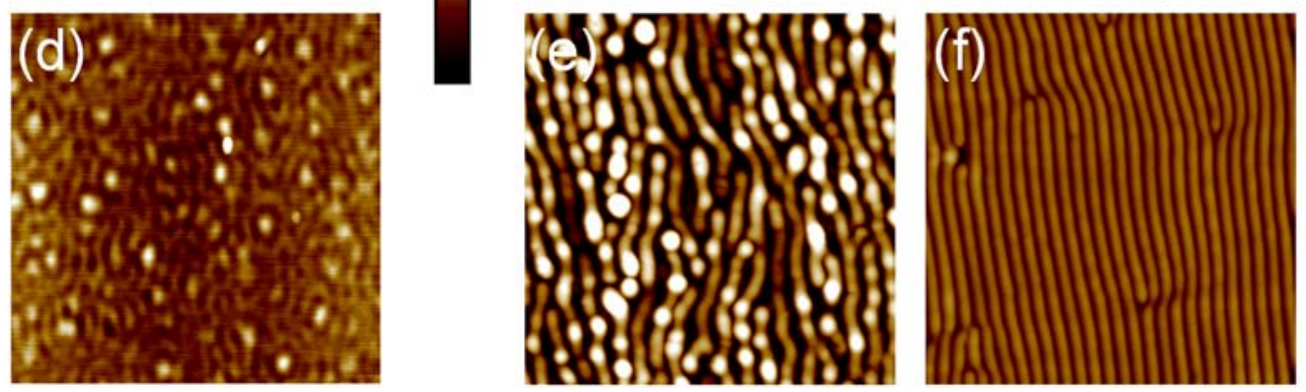

Figure 5. AFM $\left(5 \times 5 \mu \mathrm{m}^{2}\right)$ topographic images of $\operatorname{Si} 10(132 \mathrm{~nm})$ (upper row) and $\mathrm{Si} 20(278 \mathrm{~nm})$ (bottom row), irradiated at $266 \mathrm{~nm}$ with 4800 pulses and (a, d) $7 \mathrm{~mJ} / \mathrm{cm}^{2}$, (b, e) $10 \mathrm{~mJ} / \mathrm{cm}^{2}$, (f) 12 $\left.\mathrm{mJ} / \mathrm{cm}^{2}\right)$.

For a fluence of $12 \mathrm{~mJ} / \mathrm{cm}^{2}$ LIPSS obtained at the thinner film, Si10 are conspicuously disrupted (Figure 5c) indicating that this fluence is above the optimal fluence range at which LIPSS are optimally formed (Figure 5b). It is known that dealing with LIPSS in polymers when the fluence is above certain limit the whole polymer film is thermally affected and devitrification of the complete film can take place leading to dewetting and/or material ablation [9]. In the case of Si20, the irradiation at $12 \mathrm{~mJ} / \mathrm{cm}^{2}$ leads to the increase of the superficial temperature and to the heating of deeper material [13] inducing the formation of more ordered ripples (Fig. $5 \mathrm{f}$ ) in comparison to the irradiation at $10 \mathrm{~mJ} / \mathrm{cm}^{2}$ (Fig. 5 e).

In order to explain the singular behavior of the PS films with intermediate thicknesses values in the range 200-400 nm for which droplet-like entities are observed upon irradiation with 4800 pulses at $10 \mathrm{~mJ} / \mathrm{cm}^{2}$ (Figure 3d) we can consider different facts. On the one hand, the heat dissipation by the substrate of the heat generated by surface irradiation (Figure 2) is less efficient than for the thinner Si10 film, but still it plays a role since, as shown in Figure 4, a higher amount of pulses is needed in order to modify the polymer surface in comparison to thicker films. On the other hand, we can consider the optical properties of the silicon, in particular the refractive index at $266 \mathrm{~nm}$, which for silicon is higher than for PS. When the laser light reaches the silicon substrate the reflection from the substrate may introduce additional thermal effects and thus, less ordered LIPSS are expected. In fact in the case of Si20 the higher observed values of period and depth (Figure 3) suggest that the heat remains longer. Thus, a softer material exists for longer time with 
lower superficial viscosity allowing the development of ripples with larger periods. Additionally, the depth of the nanostructures in this sample is also higher, suggesting that more material is heated upon irradiation and this fact cannot be explained considering only heating by absorption of the laser light discussed in the previous paragraph. As shown in Figure 2 while for a depth of $50 \mathrm{~nm}$, which results to be the typical depth of the LIPSS obtained for most of the PS samples studied in this work, the temperature is slightly lower but similar than the one at the surface, for a depth of $280 \mathrm{~nm}$ the temperature increase is smaller than $3{ }^{\circ} \mathrm{C}$.

In order to go deeper into the role of the substrate in LIPSS formation experiments with different substrates will be discussed in the next paragraph.

\subsection{Influence of substrate on LIPSS formation}

To evaluate the role of the substrate on LIPSS formation in PS, thin films of the polymer were prepared onto silicon, glass and quartz. These three substrates were selected on the basis of their different thermal and optical properties. Again, LIPSS were prepared by irradiation with 4800 pulses at a fluence of $10 \mathrm{~mJ} / \mathrm{cm}^{2}$ of spin coated PS films from solutions with two different concentrations, $10 \mathrm{~g} / \mathrm{L}$ and $20 \mathrm{~g} / \mathrm{L}$. The topography of the nanostructured samples onto different substrates and two different thicknesses are presented in Figure 6.
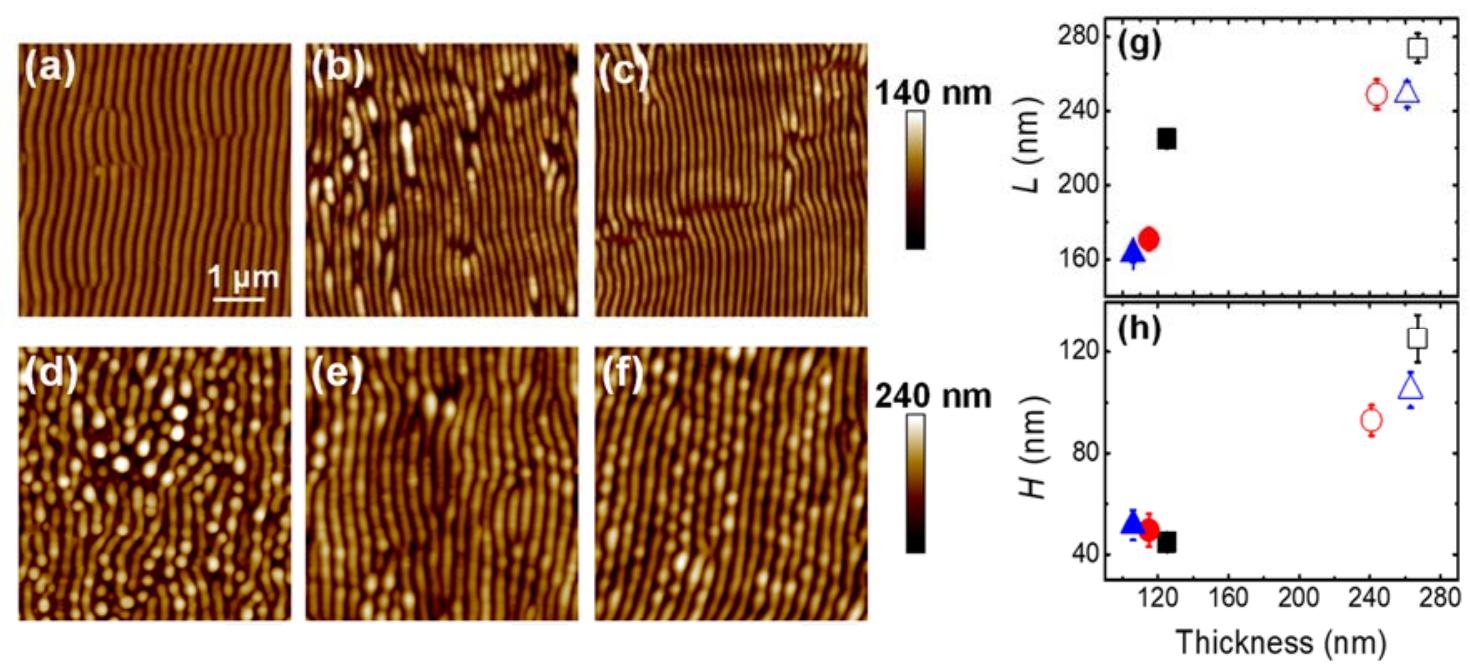

Figure 6 (Left) AFM height images $\left(5 \times 5 \mu^{2}\right)$ of PS with $10 \mathrm{~g} / \mathrm{L}$ (upper) and PS $20 \mathrm{~g} / \mathrm{L}$ (bottom) deposited on different substrates: (a, d) Silicon (Si10, Si20), (b, e) Glass (G10, G20) and (c, f) quartz (Q10, Q20). (Right) (g) Period length $(L)$ and depth $(H)$ of formed ripples for PS with $10 \mathrm{~g} / \mathrm{L}$ (filled symbols) and 20 $\mathrm{g} / \mathrm{L}$ (open symbols) deposited on different substrate: silicon $(\boldsymbol{\square})$, glass $(\boldsymbol{O})$ and quartz $(\boldsymbol{\Delta})$. Films were irradiated at $266 \mathrm{~nm}$ with a fluence $10 \mathrm{~mJ} / \mathrm{cm}^{2}$ and number of pulses 4800 . 
Figure 6 demonstrates that the formed nanostructure on the polymer surface depends on the underlying substrate. Although linear ripples are formed on film with thickness around $120 \mathrm{~nm}$ for all the substrates, different levels of order can be visualized. While the polymer on silicon, Si10, forms rather continuous and homogeneous ripples (Figure 6a), LIPSS induced in the polymer on quartz, Q10, (Figure 6c), although well-defined, appear disturbed in several places. In contrast, LIPSS generated on the polymer on glass, G10, (Figure 6b) consists of several domains, disrupted or with coalescence between ripples. At a first glance it appears that lessordered LIPSS forms on a thicker PS film of $260 \mathrm{~nm}$ in all the three substrates. However, the sample on silicon (Fig. 6d) presents a significant lower order than the others characterized by distorted lines decorated by drop-like structures. The dependence of the period and ripple depth on different substrates is presented in Figure $6 \mathrm{~g}$ and $6 \mathrm{~h}$. There is a clear influence of the nature of the substrate in the period of the LIPSS especially for thinner PS films. While LIPSS on quartz (Q10) and glass (G10) exhibit similar period values ( $\mathrm{L} \approx 170 \mathrm{~nm})$, a smaller $\mathrm{L}$ is obtained for LIPSS on silicon (Si10) $(\mathrm{L} \approx 220 \mathrm{~nm}$ ). Although weaker, a similar trend is observed for the thicker films. Substrate seems to have little effect on the ripples height in the case of thin films, but in the case of the thicker ones the depth of the LIPSS obtained on glass is smaller than the observed for silicon (Si20) and quartz (Q20).

As mentioned before, when a laser pulse reaches the polymer surface the radiation can be reflected, scattered and/or absorbed. The fraction which is absorbed by the polymer transforms into heat while the rest of the energy, if the film is thin enough, eventually may reach the underlying substrate and dissipated by it. The amount of energy at a given depth $z$ from the surface can be determined by the Beer-Lambert law: $I(z)=I_{0} e^{-\alpha z}$, where $I_{0}$ is the incident light intensity and $\alpha$ is the absorption coefficient. For instance, for a $434 \mathrm{~nm}$ thick PS sample (PS30), $93 \%$ of the incident energy is transmitted and it will reach the substrate. Thus, the thermal properties of the substrate can be relevant. The differences observed in the morphology of the LIPSS formed on PS films with different thicknesses and deposited onto different substrates may be explained on the basis of the substrate physical properties, in particular to their thermal and optical properties. The temperature increase at the substrate surface due to the transmitted light through the PS film can be estimated by using the values of the corresponding physical properties listed in Table 2 as:

$$
\Delta T=\frac{F \alpha}{c \rho}
$$

Where $\mathrm{F}$ is the fluence, $\alpha$ the absorption coefficient, $c$ the specific heat and $\rho$ the density. The estimated temperature increase has been calculated using the value of the incoming fluence and the values are reported in Table 3. 
Table 3. Temperature increase $(\Delta T)$, Thermal diffusivity $(D)$, thermal diffusion time $\left(t_{d}\right)$ and thermal diffusion length $\left(l_{S}\right)$ of the substrates

\begin{tabular}{cccc} 
& Silicon & Quartz & Glass \\
\hline $\boldsymbol{\Delta} \boldsymbol{T}(\mathbf{K})$ & $1.2 \times 10^{3}$ & $1.6 \times 10^{-5}$ & 460 \\
$\boldsymbol{D}\left(\mathbf{m}^{\mathbf{2}} \cdot \mathbf{s}^{-1}\right)$ & $9.97 \times 10^{-5}$ & $5.7 \times 10^{-6}$ & $5.15 \times 10^{-7}$ \\
$\boldsymbol{t}_{\boldsymbol{d}}(\mathbf{n s})$ & $2.6 \times 10^{-4}$ & - & 0.19 \\
\hline
\end{tabular}

After the fast increase of temperature the heat is dissipated depending on the thermal conductivity of the substrate. The thermal diffusion time, $t_{d}$, is given by $[13,55]$ :

$$
t_{d}=\frac{1}{D \alpha^{2}}
$$

Where $\mathrm{D}$ is thermal diffusivity of the material expressed by $D=\frac{\kappa}{\mathrm{c} \rho}$.

The calculated thermal diffusivity $(D)$ and thermal diffusion time $\left(t_{d}\right)$ are listed in Table 3 .

Although for silicon the temperature increment is higher than for glass, the thermal diffusivity is two orders of magnitude larger, and thus, the thermal diffusion time is shorter. In the case of quartz, it can be considered that temperature does not vary upon irradiation since the absorption at 266 $\mathrm{nm}$ is negligible. Accordingly, the mismatch in thermal conductivities between the polymer, PS, and the substrate in the case of glass and quartz, is smaller than when the substrate is silicon. This could explain the similar results obtained for irradiation of the different film thicknesses on glass and quartz. Additionally, only thermal effects need to be considered for these two substrates since their refractive index is roughly the same than the one of PS. Therefore, the reflection effects at the polymer-substrate interface are less important than in the case of the silicon substrate.

\section{CONCLUSION}

In conclusion, we have investigated the effect of the number of pulses, the film thickness and the supporting substrates properties on the quality of LIPSS formation for PS film. LIPSS can be obtained with different degrees of order by selecting both the film thickness and the substrate used for supporting the PS films. The thinner $(<200 \mathrm{~nm})$ and thicker $(>400 \mathrm{~nm})$ films develops well ordered LIPSS, because in the first case the substrate acts as a thermal heat sink and in the second one surface heating effects are more relevant. In contrast, distorted LIPSS with drop-like structures form in films with intermediate thicknesses $(200-400 \mathrm{~nm})$. The estimation of temperature upon pulse accumulation shows that a small positive offset caused by each individual pulse is present, and that a minimum number of pulses is necessary to induce changes in surface roughness and LIPSS formation. The supporting substrates can influence the LIPSS formation process. Differences observed in the fluence and number of pulses needed for the onset of morphological modifications is explained considering the differences in thermal conductivity 
between the polymer and the substrate. The quality of the LIPSS can be also affected by the energy reflected by the substrate. This suggests that the optical properties of the substrate can be an additional and relevant aspect to be considered.

\section{ACKNOWLEDGMENTS}

This work has been supported by Spanish Ministry of Economy and Competitiveness (MINECO) through projects CTQ2013-43086-P, MAT2014-59187-R, and MAT2015-66443-C02-1-R. J.C acknowledges the China Scholarship Council for funding her research (File NO.201207040083). E.R. also thanks MINECO for a Ramón y Cajal contract (RYC-2011-08069).The authors thank to J. Rodriguez from ICTP-CSIC for estimation of the thickness by confocal microscopy and J. V García Ramos for assistance during the UV-Vis spectra recording.

\section{Reference}

[1] V. Gregson, "Laser Material Processing," ed: Holland Publishing Company, Holland, 1984.

[2] C. Serrano, L. García-Fernández, J. P. Fernández-Blázquez, M. Barbeck, S. Ghanaati, R. Unger, J. Kirkpatrick, E. Arzt, L. Funk, P. Turón, and A. del Campo, "Nanostructured medical sutures with antibacterial properties," Biomaterials, vol. 52, pp. 291-300, 6// 2015.

[3] S. A. Boden and D. M. Bagnall, "Tunable reflection minima of nanostructured antireflective surfaces," Applied Physics Letters, vol. 93, p. 133108, 2008.

[4] X.-M. Li, D. Reinhoudt, and M. Crego-Calama, "What do we need for a superhydrophobic surface? A review on the recent progress in the preparation of superhydrophobic surfaces," Chemical Society Reviews, vol. 36, pp. 1350-1368, 2007.

[5] K. Sugioka, M. Meunier, and A. Piqué, Laser precision microfabrication: Springer, 2010.

[6] J. G. Fernandez, C. A. Mills, and J. Samitier, "Complex Microstructured 3D Surfaces Using Chitosan Biopolymer," Small, vol. 5, pp. 614-620, 2009.

[7] H. Schift, "Nanoimprint lithography: An old story in modern times? A review," Journal of Vacuum Science \& Technology B, vol. 26, pp. 458-480, 2008.

[8] L. J. Guo, "Nanoimprint Lithography: Methods and Material Requirements," Advanced Materials, vol. 19, pp. 495-513, 2007.

[9] E. Rebollar, M. Castillejo, and T. A. Ezquerra, "Laser induced periodic surface structures on polymer films: From fundamentals to applications," European Polymer Journal, vol. 73, pp. 162174, Dec 2015.

[10] I. Martín-Fabiani, S. Riedel, D. R. Rueda, J. Siegel, J. Boneberg, T. A. Ezquerra, and A. Nogales, "Micro- and Submicrostructuring Thin Polymer Films with Two and Three-Beam Single Pulse Laser Interference Lithography," Langmuir, vol. 30, pp. 8973-8979, 2014/07/29 2014.

[11] I. Martín-Fabiani, J. Siegel, S. Riedel, J. Boneberg, T. A. Ezquerra, and A. Nogales, "Nanostructuring Thin Polymer Films with Optical Near Fields," ACS Applied Materials \& Interfaces, vol. 5, pp. 11402-11408, 2013/11/13 2013.

[12] I. Martin-Fabiani, E. Rebollar, S. Perez, D. R. Rueda, M. C. Garcia-Gutierrez, A. Szymczyk, Z. Roslaniec, M. Castillejo, and T. A. Ezquerra, "Laser-Induced Periodic Surface Structures Nanofabricated on Poly(trimethylene terephthalate) Spin-Coated Films," Langmuir, vol. 28, pp. 7938-7945, May 222012.

[13] E. Rebollar, S. Perez, J. J. Hernandez, I. Martin-Fabiani, D. R. Rueda, T. A. Ezquerra, and M. Castillejo, "Assessment and formation mechanism of laser-induced periodic surface structures on polymer spin-coated films in real and reciprocal space," Langmuir, vol. 27, pp. 5596-606, May 32011.

[14] M. Bolle, S. Lazare, M. Le Blanc, and A. Wilmes, "Submicron periodic structures produced on 
polymer surfaces with polarized excimer laser ultraviolet radiation," Applied Physics Letters, vol. 60, pp. 674-676, 1992.

[15] M. Ivanov and P. Rochon, "Infrared-laser-induced periodic surface structure in azo-dye polymer," Applied Physics Letters, vol. 84, pp. 4511-4513, 2004.

[16] D. R. Rueda, I. Martin-Fabiani, M. Soccio, N. Alayo, F. Perez-Murano, E. Rebollar, M. C. GarciaGutierrez, M. Castillejo, and T. A. Ezquerra, "Grazing-incidence small-angle X-ray scattering of soft and hard nanofabricated gratings," Journal of Applied Crystallography, vol. 45, pp. 1038-1045, Oct 2012.

[17] E. Rebollar, J. R. Vazquez de Aldana, I. Martin-Fabiani, M. Hernandez, D. R. Rueda, T. A. Ezquerra, C. Domingo, P. Moreno, and M. Castillejo, "Assessment of femtosecond laser induced periodic surface structures on polymer films," Physical Chemistry Chemical Physics, vol. 15, pp. 1128711298, 2013.

[18] E. Rebollar, S. Perez, M. Hernandez, C. Domingo, M. Martin, T. A. Ezquerra, J. P. Garcia-Ruiz, and M. Castillejo, "Physicochemical modifications accompanying UV laser induced surface structures on poly(ethylene terephthalate) and their effect on adhesion of mesenchymal cells," Physical Chemistry Chemical Physics, vol. 16, pp. 17551-17559, 2014.

[19] M. Csete and Z. Bor, "Laser-induced periodic surface structure formation on polyethyleneterephthalate," Applied Surface Science, vol. 133, pp. 5-16, 1998.

[20] M. Csete, S. Hild, A. Plettl, P. Ziemann, Z. Bor, and O. Marti, "The role of original surface roughness in laser-induced periodic surface structure formation process on poly-carbonate films," Thin Solid Films, vol. 453-454, pp. 114-120, 2004.

[21] M. Csete, R. Eberle, M. Pietralla, O. Marti, and Z. Bor, "Attenuated total reflection measurements on poly-carbonate surfaces structured by laser illumination," Applied Surface Science, vol. 208209, pp. 474-480, 2003.

[22] D. Bäuerle, "Laser Processing and Chemistry. 2000," ed: Springer, Berlin.

[23] E. Rebollar, D. R. Rueda, I. Martin-Fabiani, A. Rodriguez-Rodriguez, M.-C. Garcia-Gutierrez, G. Portale, M. Castillejo, and T. A. Ezquerra, "In Situ Monitoring of Laser-Induced Periodic Surface Structures Formation on Polymer Films by Grazing Incidence Small-Angle X-ray Scattering," Langmuir, vol. 31, pp. 3973-3981, Apr 72015.

[24] M. Bolle and S. Lazare, "Characterization of submicrometer periodic structures produced on polymer surfaces with low - fluence ultraviolet laser radiation," Journal of Applied Physics, vol. 73, pp. 3516-3524, 1993.

[25] Ú. Prendergast, S. Kudzma, R. Sherlock, C. O'Connell, and T. Glynn, "TEM investigation of laserinduced periodic surface structures on polymer surfaces," 2007, pp. 64581V-64581V-10.

[26] S. Pérez, E. Rebollar, M. Oujja, M. Martín, and M. Castillejo, "Laser-induced periodic surface structuring of biopolymers," Applied Physics A, vol. 110, pp. 683-690, 2012.

[27] A. I. Rodríguez-Rodríguez, E. Rebollar, M. Soccio, T. A. Ezquerra, D. R. Rueda, J. V. Garcia-Ramos, M. Castillejo, and M.-C. Garcia-Gutierrez, "Laser-Induced Periodic Surface Structures on Conjugated Polymers: Poly (3-hexylthiophene)," Macromolecules, vol. 48, pp. 4024-4031, 2015.

[28] M. Csete, O. Marti, and Z. Bor, "Laser-induced periodic surface structures on different polycarbonate films," Applied Physics A, vol. 73, pp. 521-526, 2001.

[29] E. Rebollar, M. Sanz, S. Perez, M. Hernandez, I. Martin-Fabiani, D. R. Rueda, T. A. Ezquerra, C. Domingo, and M. Castillejo, "Gold coatings on polymer laser induced periodic surface structures: assessment as substrates for surface-enhanced Raman scattering," Physical Chemistry Chemical Physics, vol. 14, pp. 15699-15705, 2012.

[30] P. Slepička, A. Chaloupka, P. Sajdl, J. Heitz, V. Hnatowicz, and V. Švorčík, "Angle dependent laser nanopatterning of poly(ethylene terephthalate) surfaces," Applied Surface Science, vol. 257, pp. 6021-6025, 2011.

[31] P. Slepička, O. Neděla, J. Siegel, R. Krajcar, Z. Kolská, and V. Švorčík, "Ripple polystyrene nanopattern induced by KrF laser," Express Polymer Letters, vol. 8, pp. 459-466, 2014.

[32] P. Slepička, O. Neděla, P. Sajdl, Z. Kolská, and V. Švorčík, "Polyethylene naphthalate as an excellent candidate for ripple nanopatterning," Applied Surface Science, vol. 285, Part B, pp. 885-892, 2013.

[33] R. Krajcar, J. Siegel, P. Slepička, P. Fitl, and V. Švorčík, "Silver nanowires prepared on PET structured by laser irradiation," Materials Letters, vol. 117, pp. 184-187, 2014.

[34] R. Krajcar, R. Denk, P. Zeppenfeld, P. Slepička, and V. Švorčík, "Tuning the plasmonic behavior of metallic nanowires," Materials Letters, vol. 165, pp. 181-184, 2016.

[35] M. Štofik, A. Semerádtová, J. Malý, Z. Kolská, O. Neděla, D. Wrobel, and P. Slepička, "Direct 
immobilization of biotin on the micro-patterned PEN foil treated by excimer laser," Colloids and Surfaces B: Biointerfaces, vol. 128, pp. 363-369, 2015.

[36] J. Siegel, P. Slepička, J. Heitz, Z. Kolská, P. Sajdl, and V. Švorčík, "Gold nano-wires and nano-layers at laser-induced nano-ripples on PET," Applied Surface Science, vol. 256, pp. 2205-2209, 2010.

[37] J. Siegel, J. Heitz, A. Řezníčková, and V. Švorčík, "Preparation and characterization of fully separated gold nanowire arrays," Applied Surface Science, vol. 264, pp. 443-447, 2013.

[38] E. Rebollar, I. Frischauf, M. Olbrich, T. Peterbauer, S. Hering, J. Preiner, P. Hinterdorfer, C. Romanin, and J. Heitz, "Proliferation of aligned mammalian cells on laser-nanostructured polystyrene," Biomaterials, vol. 29, pp. 1796-1806, 2008.

[39] Y. Kalachyova, O. Lyutakov, P. Slepicka, R. Elashnikov, and V. Svorcik, "Preparation of periodic surface structures on doped poly(methyl metacrylate) films by irradiation with KrF excimer laser," Nanoscale Research Letters, vol. 9, 2014.

[40] N. Tsutsumi and A. Fujihara, "Pulsed laser induced spontaneous gratings on a surface of azobenzene polymer," Applied Physics Letters, vol. 85, pp. 4582-4584, 2004.

[41] M. Li, Q. H. Lu, J. Yin, Y. Qian, and Z. G. Wang, "Effects of post-thermal treatment on preparation of surface microstructures induced by polarized laser on polyimide film," Materials Chemistry and Physics, vol. 77, pp. 895-898, 2003.

[42] M. A. Green, "Self-consistent optical parameters of intrinsic silicon at $300 \mathrm{~K}$ including temperature coefficients," Solar Energy Materials and Solar Cells, vol. 92, pp. 1305-1310, 11// 2008.

[43] F. A. Lasagni, A. F. L. Udo Klotzbach, Michael Panzner, and a. V. Franke, "Fabrication and characterization in the micro-nano range," Advanced Structured Materials, vol. 10, pp. 361-377, 2011.

[44] E. C. Beder, C. D. Bass, and W. L. Shackleford, "Transmissivity and Absorption of Fused Quartz Between $0.22 \mu$ and $3.5 \mu$ from Room Temperature to $1500^{\circ} \mathrm{C}$," Applied Optics, vol. 10, pp. 22632268, 1971/10/01 1971.

[45] J. E. Mark, Physical properties of polymers handbook: Springer, 1996.

[46] P. Crystran Ltd, UK, . (2016, 24/02/2016). Silicon (Si). Available: http://www.crystran.co.uk/optical-materials/silicon-si

[47] P. Crystran Ltd, UK,. (2016). Quartz Crystal (SiO2). Available: http://www.crystran.co.uk/opticalmaterials/quartz-crystal-sio2

[48] P. Crystran Ltd, UK,. (2016). Optical Glass ( $-B K 7$ and others). Available: http://www.crystran.co.uk/optical-materials/optical-glass-n-bk7-and-others

[49] S. Nicola, G. Carbonara, A. Finizio, and G. Pierattini, "Measurement of the temperature dependence of quartz refractive indices," Applied Physics B, vol. 58, pp. 133-135.

[50] H. Li, "Refractive index of silicon and germanium and its wavelength and temperature derivatives," Journal of Physical and Chemical Reference Data, vol. 9, pp. 561-658, 1980.

[51] I. H. Malitson, "Interspecimen Comparison of the Refractive Index of Fused Silica*, $\dagger, "$ Journal of the Optical Society of America, vol. 55, pp. 1205-1209, 1965/10/01 1965.

[52] E. D. Palik, Handbook of optical constants of solids vol. 3: Academic press, 1998.

[53] C. M. Mate, M. F. Toney, and K. A. Leach, "Roughness of thin perfluoropolyether lubricant films: influence on disk drive technology," IEEE Transactions on Magnetics, vol. 37, pp. 1821-1823, 2001.

[54] R. Fardel, M. Nagel, T. Lippert, F. Nüesch, A. Wokaun, and B. S. Luk'yanchuk, "Influence of thermal diffusion on the laser ablation of thin polymer films," Applied Physics A, vol. 90, pp. 661-667, 2008.

[55] A. Vogel and V. Venugopalan, "Mechanisms of Pulsed Laser Ablation of Biological Tissues," Chemical Reviews, vol. 103, pp. 577-644, 2003/02/01 2003. 